\title{
THE PFAFFIAN AND THE LIE ALGEBRA HOMOLOGY OF SKEW-SYMMETRIC MATRICES
}

\author{
Jean-Louis Cathelineau
}

\begin{abstract}
An interplay between the Pfaffian and the homology groups of orthogonal Lie algebras is discussed. This clarifies the question of the stability for these homology groups. Along the way appear some twisted coinvariants of skewsymmetric matrices, which apparently have not been considered before.
\end{abstract}

\section{Introduction}

It is well known (see for example Milnor-Stasheff [16], Appendix C) that the Pfaffian appears as a specific invariant polynomial on the Lie algebra of even dimensional orthogonal groups, in relation with Euler classes and the GaussBonnet theorem. It also appears in the classical invariant theory of symplectic matrices (see [19]). In this paper, we want to discuss another aspect of the Pfaffian, related to the question of the stability of the homology groups, for orthogonal Lie algebras. In particular, we correct an assertion in [14]. Along the way, we consider some spaces of twisted coinvariants of skew-symmetric matrices which, up to now, seem to have been overlooked.

In what follows, $k$ is a field of characteristic 0 and $A$ is a commutative $k$ algebra. We denote by $s o(n, A)$ the Lie algebra over $k$, of $n \times n$ skew-symmetric matrices with entries in $A$, and by $H_{*}(s o(n, A), k)$ its homology [12]. By $O(n, k)$ we mean the group of orthogonal matrices with coefficients in $k$. Let also

$$
0 \rightarrow A \stackrel{d}{\rightarrow} \Omega_{A / k}^{1} \stackrel{d}{\rightarrow} \ldots \stackrel{d}{\rightarrow} \Omega_{A / k}^{n} \stackrel{d}{\rightarrow} \ldots
$$

denotes the de Rham complex of $A$ over $k$, where $\Omega_{A / k}^{n}$ is the space of Kähler differentials.

1.1. The orientation involution and main result. The adjoint action of $O(n, k)$ on $s o(n, A)$ induces an action on the homology groups $H_{*}(s o(n, A), k)$. Moreover, since the restricted action of the special orthogonal group $S O(n, k)$ is trivial (see Section 3, for more details), we get an action of $O(n, k) / S O(n, k)=$ $\{ \pm 1\}$ on $H_{*}(s o(n, A), k)$. This last action is non trivial in general : note that, contrary to $S O(n, k)$, the group $O(n, k)$ is not connected as an algebraic group.

Received September 11, 2003.

2000 Mathematics Subject Classification. 18G, 17B, 13D, 15A72.

Key words and phrases. Pfaffian, Lie algebra homology, invariant theory, cyclic and dihedral homology. 
To the +1 and -1 eigenvalues of the corresponding involution, is associated a decomposition into eigenspaces written, in what follows,

$$
H_{i}(\operatorname{so}(n, A), k)=H_{i}(\operatorname{so}(n, A), k)^{+} \oplus H_{i}(\operatorname{so}(n, A), k)^{-} .
$$

We remark that the projections identify respectively these eigenspaces to coinvariants for two actions of $O(n, k)$ on $H_{i}(\operatorname{so}(n, A), k)$. Namely the one induced by the adjoint action on the Chevalley-Eilenberg complex and the same one twisted by the determinant (see Sections 2 and 3). We will argue on these eigenspaces, as if they would be coinvariants.

Our main result is the following.

Theorem 1.1. Let $A$ be a commutative $k$-algebra, we have

i) For all $i \geq 0, H_{i}(s o(2 m+1, A), k)^{-}=0$.

ii) For $m>i, H_{i}(s o(2 m, A), k)^{-}=0$.

iii) There is a canonical isomorphism, related to the Pfaffian

$$
H_{m}(s o(2 m, A), k)^{-} \stackrel{\cong}{\rightarrow} \Omega_{A / k}^{m-1} / d \Omega_{A / k}^{m-2} .
$$

iv) For $n \geq i+1$, the natural map

$$
H_{i}(\operatorname{so}(n, A), k)^{+} \rightarrow H_{i}(\operatorname{so}(n+1, A), k)^{+}
$$

is an isomorphism.

v) For $n \geq 2 i+1$, the natural map

$$
H_{i}(\operatorname{so}(n, A), k) \rightarrow H_{i}(\operatorname{so}(n+1, A), k)
$$

is an isomorphism.

As we will see in Proposition 3.4, the isomorphism in iii) can be made explicit in term of the polarization of the Pfaffian. Assertion iv) is actually proved in [14] (see Theorem 7.1), but, in this paper as well as in [12] Chapter 10, it is wrongly stated for the groups $H_{i}(s o(n, A), k)$, instead of $H_{i}(s o(n, A), k)^{+}$. Assertion v) is a direct consequence of ii) and iv).

As a corollary, we see that the kernel of the surjective map $H_{m}(s o(2 m, A), k) \rightarrow$ $H_{m}(s o(2 m+1, A), k)$, is isomorphic to $\Omega_{A / k}^{m-1} / d \Omega_{A / k}^{m-2}$.

It is likely that the theorem generalizes, to cover the more general case considered in [14], where $A$ is a non-commutative unital algebra with involution. But the proof given in what follows does not work simply as it is, in the more general situation.

1.2. Analogy. The first three assertions in Theorem 1.1 present similarities with some results proved in $[5,6]$, for the homology of orthogonal groups. To explain the analogy, replace the pair $(A, k)$ by $(F, \mathbb{Q})$, where $F$ is a field of characterisitic zero. Let $H_{i}\left(O(n, F), \mathbb{Q}^{t}\right)$ denote the homology of the orthogonal group $O(n, F)$ with twisted coefficients, where $t$ means the twisted action of $O(n, F)$ by the determinant. The groups $H_{i}(s o(n, A), k)^{+}$and $H_{i}(s o(n, A), k)^{-}$are respectively the counterparts of $H_{i}(O(n, F), \mathbb{Q})$ and $H_{i}\left(O(n, F), \mathbb{Q}^{t}\right)$. Indeed let $H_{i}(S O(n, F), \mathbb{Q})^{+} \oplus H_{i}(S O(n, F), \mathbb{Q})^{-}$be the decomposition of $H_{i}(S O(n, F), \mathbb{Q})$, 
under the involution arising from the conjugation action of $O(n, F)$ on $S O(n, F)$. From the Hochschild-Serre spectral sequences with coefficients (see [3]), for the extension

$$
0 \rightarrow S O(n, F) \rightarrow O(n, F) \stackrel{\text { det }}{\longrightarrow}\{ \pm 1\} \rightarrow 0,
$$

we get isomorphisms

$$
\begin{aligned}
& H_{i}(S O(n, F), \mathbb{Q})^{+} \cong H_{i}(O(n, F), \mathbb{Q}), \\
& H_{i}(S O(n, F), \mathbb{Q})^{-} \cong H_{i}\left(O(n, F), \mathbb{Q}^{t}\right) .
\end{aligned}
$$

Now it was proved in $[6]$ that $H_{i}\left(O(n, F), \mathbb{Q}^{t}\right)=0$ for $n$ odd, and

$$
H_{i}\left(O(2 m, F), \mathbb{Q}^{t}\right)=0 \text { for } i<m \text {. }
$$

It was also proved that for $F$ algebraically closed, $H_{m}\left(O(2 m, F), \mathbb{Q}^{t}\right)$ is isomorphic to the Milnor $K$-theory group $K_{m}^{M}(F) \otimes \mathbb{Q}$. But we know that, thinking of cyclic homology as additive Quillen $K$-theory, the counterpart of $K_{m}^{M}(F) \otimes \mathbb{Q}$ is given by $\Omega_{F / \mathbb{Q}}^{m-1} / d \Omega_{F / \mathbb{Q}}^{m-2}$.

In view of the previous analogy, the following stability conjecture for the homology groups of $O(n, F)$ and $S O(n, F)$ is plausible, for $F$ an algebraically closed field of characteristic 0 .

"The natural map

$$
H_{i}(O(n, F), \mathbb{Q}) \rightarrow H_{i}(O(n+1, F), \mathbb{Q})
$$

is an isomorphism, for $n \geq i+1$.

Moreover, the natural map

$$
H_{i}(S O(n, F), \mathbb{Q}) \rightarrow H_{i}(S O(n+1, F), \mathbb{Q})
$$

is an isomorphism, for $n \geq 2 i+1$, and the map

$$
H_{m}(S O(2 m, F), \mathbb{Q}) \rightarrow H_{m}(S O(2 m+1, F), \mathbb{Q})
$$

is onto, with kernel isomorphic to $K_{m}^{M}(F) \otimes \mathbb{Q}$."

I don't think such a precise result has already been proved. We refer the interested reader to $[7,18,21,17,20]$, for various known results concerning the stability of the homology of orthogonal groups.

1.3. Illustration. We want to notice a few facts already known, which fit with Theorem 1.1, and show that the homology of $s o(n, A)$ can be tricky. We denote respectively by $H C_{*}(A)$ and $H D_{*}(A)$ the cyclic and dihedral homology of $A$, as a $k$-algebra (see $[12])$.

1) For $s o(2, A)$, it is straightforward that

$$
\begin{gathered}
H_{*}(s o(2, A), k)^{+}=\oplus_{l \geq 0} H_{2 l}(s o(2, A), k)=\oplus_{l \geq 0} \wedge_{k}^{2 l} A, \\
H_{*}(s o(2, A), k)^{-}=\oplus_{l \geq 0} H_{2 l+1}(s o(2, A), k)=\oplus_{l \geq 0} \wedge_{k}^{2 l+1} A .
\end{gathered}
$$

2) It is known from results in Kassel [10] (see also [4]) that, for $n=3$ and $n \geq 5$, 


$$
H_{2}(\operatorname{so}(n, A), k)=H_{2}(s o(n, A), k)^{+} \cong H C_{1}(A) \cong \Omega_{A / k}^{1} / d A .
$$

The case of the non-simple algebra $s o(4, A) \cong s o(3, A) \times s o(3, A)$ is special. We get from the Künneth formula, since $H_{1}(s o(3, A), k)=0$,

$$
H_{2}(s o(4, A), k) \cong H_{2}(s o(3, A), k) \times H_{2}(s o(3, A), k) \cong \Omega_{A / k}^{1} / d A \times \Omega_{A / k}^{1} / d A .
$$

One can see that the involution exchanges the two factors. This implies that

$$
H_{2}(s o(4, A), k)^{+} \cong H_{2}(s o(4, A), k)^{-} \cong H C_{1}(A) \cong \Omega_{A / k}^{1} / d A .
$$

3) The case of $H_{3}$ is interesting. As a consequence of [4] Théorème 1 , we have ${ }^{1}$

$$
H_{3}(\operatorname{so}(n, A), k) \cong H D_{2}(A), \text { for } n=3,5, \text { and } n>6,
$$

and

$$
H_{3}(s o(6, A), k) \cong H C_{2}(A) .
$$

The reason why this last case is exceptional comes in [4] from the fact that $s o(6)$ is of simple type $D_{3}=A_{3}$.

Moreover for $n=4$, the Künneth formula implies

$$
H_{3}(s o(4, A), k)^{+} \cong H_{3}(s o(4, A), k)^{-} \cong H D_{2}(A) .
$$

We conclude that the decompositions of $H_{3}(s o(n, A), k)$ are as follows.

i) For $n=3,5, n>6$,

$$
H_{3}(\operatorname{so}(n, A), k)^{+} \oplus H_{3}(s o(n, A), k)^{-} \cong H D_{2}(A) \oplus 0 .
$$

ii) For $n=4$,

$$
H_{3}(s o(4, A), k)^{+} \oplus H_{3}(s o(4, A), k)^{-} \cong H D_{2}(A) \oplus H D_{2}(A) .
$$

iii) For $n=6$,

$$
H_{3}(s o(6, A), k)^{+} \oplus H_{3}(s o(6, A), k)^{-} \cong H D_{2}(A) \oplus \Omega_{A / k}^{2} / d \Omega_{A / k}^{1} .
$$

Note that these calculations were already in contradiction with the stability result in [14].

We recall that for a smooth $k$-algebra $A, H D_{2}(A) \cong \operatorname{Ker}\left(A \stackrel{d}{\rightarrow} \Omega_{A / k}^{1}\right)$. In particular for $A=\mathbb{C}, k=\mathbb{Q}$, we get the algebraic numbers.

\footnotetext{
${ }^{1}$ I take the opportunity to correct the sign for the dihedral involution in [4] which has to be read $n(n-1) / 2$, in accordance with the one in the book [12].
} 


\section{Twisted invariants of matrices}

2.1. The polarized Pfaffian. The Pfaffian (see Bourbaki [2], 5.2)

$$
\text { Pf }: s o(2 m, k) \rightarrow k,
$$

is the following polynomial, in the entries $a_{i, j}$ of a skew-symmetric $2 m \times 2 m$ matrix $M$, where $S_{n}$ is the symmetric group.

$$
\operatorname{Pf}(M)=\sum_{\substack{\sigma \in S_{2 m} \\ \sigma(2 i+1)<\sigma(2 i+2) \\ \sigma(1)<\sigma(3)<\ldots<\sigma(2 m-1)}} \epsilon_{\sigma} a_{\sigma(1), \sigma(2)} a_{\sigma(3), \sigma(4)} \ldots a_{\sigma(2 m-1), \sigma(2 m)} .
$$

We recall the following property which is crucial in what follows. For any $2 m \times 2 m$ matrix $B$, with coefficients in $k$,

$$
P f\left(B M^{t} B\right)=\operatorname{det} B \cdot P f(M),
$$

where ${ }^{t} B$ means the transpose matrix. In particular if $B$ is an orthogonal matrix

$$
P f\left(B M B^{-1}\right)=\operatorname{det} B \cdot P f(M) .
$$

We express this fact by saying that $P f$ is a twisted invariant, for the adjoint action of $O(2 m, k)$ in $s o(2 m, k)$. More generally :

Definition 2.1. A multilinear map

$$
\Phi: s o(n, k)^{l} \rightarrow k,
$$

is called a twisted invariant, if for any orthogonal matrix $B \in O(n, k)$ and matrices $M_{i} \in \operatorname{so}(n, k)$ :

$$
\Phi\left(B M_{1} B^{-1}, B M_{2} B^{-1}, \ldots, B M_{l} B^{-1}\right)=\operatorname{det} B . \Phi\left(M_{1}, M_{2}, \ldots, M_{l}\right) .
$$

We call polarized Pfaffian, the polarization $\tilde{P f}$ of the Pfaffian defined as follows : $\tilde{P f}\left(M_{1}, M_{2}, \ldots, M_{m}\right)$ is the coefficient of $t_{1} t_{2} \ldots t_{m}$ in the expansion of $\operatorname{Pf}\left(M_{1} t_{1}+\right.$ $\left.M_{2} t_{2}+\ldots+M_{m} t_{m}\right)$, where $t_{1}, \ldots, t_{m}$ are indeterminates, and $M_{i} \in s o(2 m, k)$ for $i=1, \ldots, m$.

Proposition 2.2. The polarized Pfaffian $\tilde{P f}$ satisfies the following properties :

i) It is a twisted invariant of $m$ skew-symmetric matrices and is symmetric as a multilinear form $\mathrm{so}(2 m, k)^{m} \rightarrow k$.

ii) It is given by the explicit formula

$$
\tilde{P f}\left(M_{1}, M_{2}, \ldots, M_{m}\right)=\sum_{\substack{\sigma \in S_{2 m} \\ \sigma(2 i+1)<\sigma(2 i+2)}} \epsilon_{\sigma} a_{\sigma(1), \sigma(2)}^{1} a_{\sigma(3), \sigma(4)}^{2} \ldots a_{\sigma(2 m-1), \sigma(2 m)}^{m},
$$

where $M_{l}=\left(a_{i, j}^{l}\right), l=1, \ldots, m$.

iii) $\operatorname{Pf}(M)=1 / m ! \tilde{P} f(M, M, \ldots, M)$. 
Let $F_{i, j}, 1 \leq i<j \leq n$, be the skew-symmetric matrix with coefficient 1 (resp. -1 ) at the $(i, j)$ (resp. $(j, i))$ position, and 0 elsewhere. Of course, these matrices form a basis of $s o(n, k)$ over $k$. From the formula in ii) Proposition 2.2, we get

$$
\tilde{P f}\left(F_{1,2}, F_{3,4}, \ldots, F_{2 m-1,2 m}\right)=1 \text {. }
$$

2.2. Twisted invariants and coinvariants of skew-symmetric matrices. Consider the adjoint action of $O(n, k)$ on $\otimes^{l} s o(n, k)$, given by

$$
\text { B. } M_{1} \otimes \ldots \otimes M_{l}=B M_{1} B^{-1} \otimes \ldots \otimes B M_{l} B^{-1},
$$

for $B \in O(n, k)$. This action of $O(n, k)$ factorizes to an action of

$$
O(n, k) / S O(n, k)=\{ \pm 1\}
$$

on the space of coinvariants $\left(\otimes^{l} s o(n, k)\right)_{S O(n, k)}$. The associated involution induces a decomposition

$$
\left(\otimes^{l} s o(n, k)\right)_{S O(n, k)}=\left(\otimes^{l} s o(n, k)\right)_{S O(n, k)}^{+} \oplus\left(\otimes^{l} s o(n, k)\right)_{S O(n, k)}^{-} .
$$

Note that $\left(\otimes^{l} s o(n, k)\right)_{S O(n, k)}^{+} \cong\left(\otimes^{l} s o(n, k)\right)_{O(n, k)}$. This space has been a classical object of study in invariant theory $[19,12]$. But we insist on the fact that the equality

$$
\left(\otimes^{l} s o(n, k)\right)_{S O(n, k)}=\left(\otimes^{l} s o(n, k)\right)_{O(n, k)},
$$

is not always true, as the two following propositions show.

We call twisted coinvariants the elements of $\left(\otimes^{l} s o(n, k)\right)_{S O(n, k)}^{-}$: actually in what follows, we indentify $\left(\otimes^{l} s o(n, k)\right)_{S O(n, k)}^{-}$with the coinvariants of $\otimes^{l} s o(n, k)$ $=\otimes^{l} s o(n, k) \otimes k$, under the diagonal action $O(n, k)$, by the adjoint action on $s o(n, k)$ and by the product by the determinant on $k$.

Proposition 2.3. The polarized Pfaffian

$$
\tilde{P f}: \operatorname{so}(2 m, k)^{m} \rightarrow k,
$$

induces an isomorphism

$$
\left(\otimes^{m} s o(2 m, k)\right)_{S O(2 m, k)}^{-} \stackrel{\cong}{\rightrightarrows} k .
$$

Any twisted invariant of $m$ matrices in $s o(2 m, k)$ is a multiple of the polarized Pfaffian.

Proof: As a twisted invariant, the polarized Pfaffian $\tilde{P f}$ clearly induces a non trivial linear map

$$
\left(\otimes^{m} s o(2 m, k)\right)_{S O(2 m, k)}^{-} \rightarrow k .
$$

We have just to prove that $\left(\otimes^{m} s o(2 m, k)\right)_{S O(2 m, k)}^{-}$is generated by the class of $F_{1,2} \otimes F_{3,4} \otimes \ldots \otimes F_{2 m-1,2 m}$ (recall that we consider $\left(\otimes^{m} s o(2 m, k)\right)_{S O(2 m, k)}^{-}$as a space of coinvariants, that is as a quotient). 
For this, we first look at the tensors $F_{i_{1}, i_{2}} \otimes F_{i_{3}, i_{4}} \otimes \ldots \otimes F_{i_{2 m-1}, i_{2 m}}$, such that $\left\{i_{1}, i_{2}, \ldots, i_{2 m}\right\}=\{1,2,3, \ldots, 2 m\}$. For such a tensor, we have in $\left(\otimes^{m} s o(2 m, k)\right)_{S O(2 m, k)}^{-}$, the relation

$$
F_{i_{1}, i_{2}} \otimes F_{i_{3}, i_{4}} \otimes \ldots \otimes F_{i_{2 m-1}, i_{2 m}}=\epsilon_{\sigma} \cdot F_{1,2} \otimes F_{3,4} \otimes \ldots \otimes F_{2 m-1,2 m} .
$$

We have noted $\epsilon_{\sigma}$ the signature of the permutation

$$
1 \mapsto i_{1}, 2 \mapsto i_{2}, \ldots, 2 m \mapsto i_{2 m} .
$$

This equality comes from the adjoint action of the corresponding permutation matrix.

On the other hand, let $F_{i_{1}, i_{2}} \otimes F_{i_{3}, i_{4}} \otimes \ldots \otimes F_{i_{2 m-1}, i_{2 m}}$, with $\left\{i_{1}, i_{2}, \ldots, i_{2 m}\right\} \neq$ $\{1,2, \ldots, 2 m\}$. Again, up to conjugation by a permutation matrix, we can assume that $\left\{i_{1}, i_{2}, \ldots, i_{2 m}\right\} \subset\{1,2, \ldots, 2 m-1\}$. But then the adjoint action by the diagonal matrix $(1,1, \ldots, 1,-1)$, of determinant -1 , shows that in $\otimes^{m} s o(2 m, k)_{S O(2 m, k)}^{-}$

$$
\text { 2. } F_{i_{1}, i_{2}} \otimes F_{i_{3}, i_{4}} \otimes \ldots \otimes F_{i_{2 m-1}, i_{2 m}}=0 .
$$

This achieves the proof of the proposition.

Here is another simple result :

Proposition 2.4. We have

i) For $n$ odd and any $l,\left(\otimes^{l} \operatorname{so}(n, k)\right)_{S O(n, k)}^{-}=0$, that is

$$
\left(\otimes^{l} s o(n, k)\right)_{S O(n, k)}=\left(\otimes^{l} s o(n, k)\right)_{O(n, k)} .
$$

ii) For $l<m$

$$
\left(\otimes^{l} s o(2 m, k)\right)_{S O(2 m, k)}^{-}=0 .
$$

iii) For $l \geq m$, the space $\left(\otimes^{l} s o(2 m, k)\right)_{S O(2 m, k)}^{-}$is non trivial.

For the proof of part i) we remark that, if $n$ is odd, the action of

$$
O(n, k) / S O(n, k) \text { on }\left(\otimes^{l} s o(n, k)\right)_{S O(n, k)}
$$

is trivial, since it is represented by the adjoint action of the matrix $-I d$.

For ii), we procceed as in the end of the proof of Proposition 2.2.

Finally, for $l \geq m$ in iii), we can consider, for example, the following twisted multilinear invariant of $l$ skew-symmetric $2 m \times 2 m$ matrices, derived from Lie brackets,

$$
\begin{gathered}
\left(\otimes^{l} s o(2 m, k)\right)_{S O(2 m, k)}^{-} \rightarrow k \\
\left(M_{1}, M_{2}, \ldots, M_{l}\right) \mapsto \tilde{P f}\left(M_{1}, \ldots, M_{m-1},\left[\left[\ldots\left[\left[M_{m}, M_{m+1}\right], M_{m+2}\right], \ldots, M_{l-1}\right], M_{l}\right]\right) .
\end{gathered}
$$

One check easily that this invariant is non trivial. 


\section{Proof of Theorem 1.1}

Let us consider the coinvariants : $\left(\wedge_{k}^{l} s o(n, A)\right)_{S O(n, k)}$. Proceeding as in [15], we have a canonical isomorphism

$$
\left(\wedge_{k}^{l} s o(n, A)\right)_{S O(n, k)} \cong\left(\left(\otimes^{l} s o(n, k)\right)_{S O(n, k)} \otimes\left(\otimes^{l} A\right)\right)_{S_{l}},
$$

where the action of the symmetric group $S_{l}$ is twisted by the signature.

Proposition 3.1. The map

$$
\left(M_{1} \otimes \ldots \otimes M_{m}\right) \otimes\left(a_{1} \otimes \ldots \otimes a_{m}\right) \mapsto \tilde{P f}\left(M_{1}, \ldots, M_{m}\right) a_{1} \wedge \ldots \wedge a_{m},
$$

induces an isomorphism

$$
\left(\wedge_{k}^{m} s o(2 m, A)\right)_{S O(2 m, k)}^{-}=\left(\left(\otimes^{m} s o(2 m, k)\right)_{S O(2 m, k)}^{-} \otimes\left(\otimes^{m} A\right)\right)_{S_{m}} \cong \wedge_{k}^{m} A .
$$

This comes from the symmetry of the polarized Pfaffian as a multilinear form and the fact that $S_{m}$ acts trivially on $\left(\otimes^{m} s o(2 m, k)\right)_{S O(2 m, k)}$, by Proposition 2.3. The inverse of this isomorphism reads

$$
a_{1} \wedge \ldots \wedge a_{m} \mapsto F_{1,2}^{a_{1}} \otimes \ldots \otimes F_{2 m-1,2 m}^{a_{m}},
$$

where for $i<j, F_{i, j}^{a} \in s o(2 m, A)$ is defined as $a F_{i, j}$. For $i>j$, we will also put $F_{i, j}^{a}=-F_{j, i}^{a}=F_{j, i}^{-a}$, in what follows.

Recall that the adjoint action of $s o(n, k)$ on $\wedge^{l} s o(n, A)$ is given by

$$
\text { C. } U_{1} \wedge \ldots \wedge U_{l}=\sum_{i=1}^{i=l} U_{1} \wedge \ldots \wedge\left[C, U_{i}\right] \wedge \ldots \wedge U_{l},
$$

for $C \in \operatorname{so}(n, k)$ and $U_{i} \in \operatorname{so}(n, A)$.

Lemma 3.2. We have the equalities between spaces of coinvariants under adjoint actions

$$
\begin{aligned}
& \left(\wedge^{l} s o(n, k)\right)_{s o(n, k)}=\left(\wedge^{l} s o(n, k)\right)_{S O(n, k) .} . \\
& \left.\left(\wedge_{k}^{l} s o(n, A)\right)_{s o(n, k)}=\wedge_{k}^{l} s o(n, A)\right)_{S O(n, k) .} .
\end{aligned}
$$

Proof : Actually $\left(\otimes^{l} s o(n, k)\right)_{s o(n, k)}=\left(\otimes^{l} s o(n, k)\right)_{S O(n, k)}$, because $S O(n, k)$ is a connected algebraic group, and $k$ is of characteristic zero (see [1,9]).

The classical Chevalley-Eilenberg $[8,11]$ complex calculating the homology of so $(n, A)$ reads

$$
\ldots \rightarrow \wedge_{k}^{l} s o(n, A) \stackrel{d}{\rightarrow} \wedge_{k}^{l-1} s o(n, A) \rightarrow \ldots \rightarrow s o(n, A) \rightarrow k \rightarrow 0,
$$

with differential $d$ given by

$$
d\left(u_{1} \wedge \ldots \wedge u_{l}\right)=\sum_{i<j}(-1)^{i+j-1}\left[u_{i}, u_{j}\right] \wedge u_{1} \wedge \ldots \wedge \widehat{u_{i}} \wedge \ldots \wedge \widehat{u_{j}} \wedge \ldots \wedge u_{l} .
$$

As in [15], Proposition 6.4, since $s o(n, k)$ is semi-simple, this complex is quasiisomorphic to the complex of coinvariants

$$
\ldots \rightarrow\left(\wedge_{k}^{l} s o(n, A)\right)_{s o(n, k)} \stackrel{d}{\rightarrow}\left(\wedge_{k}^{l-1} s o(n, A)\right)_{s o(n, k)} \rightarrow \ldots \rightarrow s o(n, A)_{s o(n, k)} \rightarrow k \rightarrow 0 .
$$


Now since 2 is invertible in $k$, we find that the complex

$$
\ldots \rightarrow\left(\wedge_{k}^{l} s o(n, A)\right)_{s o(n, k)}^{-} \stackrel{d}{\rightarrow}\left(\wedge_{k}^{l-1} \operatorname{so}(n, A)\right)_{s o(n, k)}^{-} \rightarrow \ldots \rightarrow s o(n, A)_{s o(n, k)}^{-} \rightarrow 0 .
$$

computes the homology groups $H_{*}(s o(n, A), k)^{-}$.

From Proposition 2.4 and Lemma 3.2, we get immediatly the parts i) and ii) of Theorem 1.1. We have already observed that iv) is implicit in [14], and that $\mathrm{v})$ is a byproduct. Then it remains to prove the point iii).

We recall from [15] Section 6, that the map

$$
\begin{gathered}
\wedge_{k}^{m} A \rightarrow \Omega_{A / k}^{m-1} / d \Omega_{A / k}^{m-2} \\
a_{1} \wedge a_{2} \wedge \ldots \wedge a_{m} \mapsto a_{1} d a_{2} \ldots d a_{m},
\end{gathered}
$$

induces an isomorphism between $\Omega_{A / k}^{m-1} / d \Omega_{A / k}^{m-2}$ and the quotient of $\wedge_{k}^{m} A$ by the subspace generated by the elements

$$
\left(a_{1} a_{2} \wedge a_{3}-a_{1} \wedge a_{2} a_{3}+a_{1} a_{3} \wedge a_{2}\right) \wedge a_{4} \wedge \ldots \wedge a_{m+1} .
$$

Now since $\left(\wedge_{k}^{m-1} s o(2 m, A)\right)_{s o(2 m, k)}^{-}=0$ by Proposition 2.4, we have just to prove the following lemma.

Lemma 3.3. Through the isomorphism of Proposition 3.1, the image of the differential

$$
d:\left(\wedge_{k}^{m+1} s o(2 m, A)\right)_{s o(2 m, k)}^{-} \rightarrow\left(\wedge_{k}^{m} s o(2 m, A)\right)_{s o(2 m, k)}^{-}
$$

is the subspace of $\wedge_{k}^{m} A$ generated by the elements

$$
\left(a_{1} a_{2} \wedge a_{3}-a_{1} \wedge a_{2} a_{3}+a_{1} a_{3} \wedge a_{2}\right) \wedge a_{4} \wedge \ldots \wedge a_{m} .
$$

Proof: Note first that we have the commutation relations : $\left[F_{i, j}^{a}, F_{i, j}^{b}\right]=0$, $\left[F_{i, j}^{a}, F_{k, l}^{b}\right]=0$, for $i, j, k, l$ distinct and $\left[F_{i, j}^{a}, F_{i, l}^{b}\right]=-F_{j, l}^{a b}$, for $i, j, l$ distinct. By using these commutation relations and actions by permutation matrices, one can check that all the generators

$$
F_{i_{1}, i_{2}}^{a_{1}} \wedge \ldots \wedge F_{i_{2 m+1}, i_{2 m+2}}^{a_{m+1}}
$$

of $\left(\wedge_{k}^{m+1} s o(2 m, A)\right)_{s o(2 m, k)}^{-}$, which are not of the type

$$
F_{1,2}^{a_{1}} \wedge F_{1,3}^{a_{2}} \wedge F_{1,4}^{a_{3}} \wedge F_{5,6}^{a_{4}} \wedge F_{7,8}^{a_{5}} \wedge \ldots \wedge F_{2 m-1,2 m}^{a_{m+1}},
$$

are either 0 or of zero differential.

Now using again invariance by permutation matrices and commutation relations, we get

$$
\begin{aligned}
& d\left(F_{1,2}^{a_{1}} \wedge F_{1,3}^{a_{2}} \wedge F_{1,4}^{a_{3}} \wedge F_{5,6}^{a_{4}} \wedge \ldots \wedge F_{2 m-1,2 m}^{a_{m+1}}\right)= \\
& =\left(-F_{2,3}^{a_{1} a_{2}} \wedge F_{1,4}^{a_{3}}+F_{2,4}^{a_{1} a_{3}} \wedge F_{1,3}^{a_{2}}-F_{3,4}^{a_{2} a_{3}} \wedge F_{1,2}^{a_{1}}\right) \wedge F_{5,6}^{a_{4}} \wedge \ldots \wedge F_{2 m-1,2 m}^{a_{m+1}} \\
& \quad=\left(-F_{1,2}^{a_{1} a_{2}} \wedge F_{3,4}^{a_{3}}-F_{1,2}^{a_{1} a_{3}} \wedge F_{3,4}^{a_{2}}+F_{1,2}^{a_{1}} \wedge F_{3,4}^{a_{2} a_{3}}\right) \wedge F_{5,6}^{a_{4}} \wedge \ldots \wedge F_{2 m-1,2 m}^{a_{m+1}} .
\end{aligned}
$$

This proves the lemma and completes the proof of Theorem 1.1.

From the previous discussion, we eventually get the following. 
Proposition 3.4. The isomorphism

$$
H_{m}(s o(2 m, A), k)^{-} \cong \Omega_{A / k}^{m-1} / d \Omega_{A / k}^{m-2}
$$

is induced by the map

$$
\left(M_{1} \otimes \ldots \otimes M_{m}\right) \otimes\left(a_{1} \otimes \ldots \otimes a_{m}\right) \mapsto \tilde{P} f\left(M_{1}, \ldots, M_{m}\right) a_{1} d a_{2} \ldots d a_{m},
$$

where $M_{i} \in \operatorname{so}(2 m, k)$.

\section{Complements}

We give a few complements without details.

4.1. Connection with obstruction to stability for the homology of $g l(m, A)$. Recall that by [15], there is a natural isomorphism

$$
H_{m}(g l(m, A), k) / \operatorname{Im} H_{m}(g l(m-1, A), k) \cong \Omega_{A / k}^{m-1} / d \Omega_{A / k}^{m-2},
$$

where $\operatorname{Im} H_{m}(g l(m-1, A), k)$ is the image of the stabilization map : $H_{m}(g l(m-$ $1, A), k) \rightarrow H_{m}(g l(m, A), k)$. If -1 is a square in $k$, the Lie algebra $s o(2 m, A)$ is naturally isomorphic to the Lie algebra $s o(m, m, A)$ of $m \times m$-matrices $\left(\begin{array}{cc}A & B \\ C & D\end{array}\right)$, such that : $B, C$ are skew-symmetric, and $D=-{ }^{t} A$. Through this isomorphism, one can consider the natural map

$$
H_{m}(g l(m, A), k) \rightarrow H_{m}(s o(2 m, A), k)^{-},
$$

arising from the morphism of Lie algebras

$$
\begin{gathered}
g l(m, A) \rightarrow \operatorname{so}(m, m, A) \\
M \mapsto\left(\begin{array}{cc}
M & 0 \\
0 & -{ }^{t} M
\end{array}\right) .
\end{gathered}
$$

Since $H_{m}(s o(2 m-1, A), k)^{-}=0$, this map induces, by naturality, a map

$$
H_{m}(g l(m, A), k) / \operatorname{Im} H_{m}(g l(m-1, A), k) \rightarrow H_{m}(s o(2 m, A), k)^{-} .
$$

This last map is an isomorphism.

4.2. One question. We observe that $\Omega_{A / k}^{m-1} / d \Omega_{A / k}^{m-2}$ is the piece $H C_{m-1}^{(m-1)}(A)$ of the cyclic homology group $H C_{m-1}(A)$, in the so-called Adams decomposition of $[13,12]$. On the other hand, $H D_{2}(A)$ is the piece $H C_{2}^{(1)}(A)$ of $H C_{2}(A)$. Thus we have

$$
H_{m}(s o(2 m, A), k)^{-} \cong H C_{m-1}^{(m-1)}(A)
$$

for any $m$, and

$$
H_{m+1}(s o(2 m, A), k)^{-} \cong H C_{m}^{(m-1)}(A)
$$

for $m \leq 2$.

Is there any relation in general, between the group $H_{m+1}(s o(2 m, A), k)^{-}$and the piece $H C_{m}^{(m-1)}(A)$ of the cyclic homology group $H C_{m}(A)$ ? 
4.3. General remarks on twisted invariants. The classification of twisted invariants and coinvariants of skew-symmetric matrices seems to be an interesting problem. Of course, one can construct quite a lot of twisted invariants, combining the polarized Pfaffian and the Lie brackets. For example there is, up to a scalar, a unique twisted invariant

$$
s o(4, k)^{3} \rightarrow k,
$$

which is skew-symmetric as a multilinear form. It is given by

$$
\left(M_{1}, M_{2}, M_{3}\right) \mapsto \tilde{P f}\left(\left[M_{1}, M_{2}\right], M_{3}\right) .
$$

Concerning the twisted coinvariants, we note that the natural maps

$$
\left(\otimes^{l} s o(2 m, k)\right)_{S O(2 m, k)}^{-} \rightarrow\left(\otimes^{l} s o(2 m+2, k)\right)_{S O(2 m, k)}^{-}
$$

are always zero, by Proposition $2.4 \mathrm{i}$ ), since they factorize through the space $\left(\otimes^{l} s o(2 m+1, k)\right)_{S O(2 m+1, k)}^{-}$.

However, one can consider the space

$$
\bigoplus_{l, m ; l \leq m}\left(\otimes^{l} s o(2 m, k)\right)_{S O(2 m, k)}^{-}
$$

as a whole. This space has a natural structure of bigraded $k$-algebra. The product is given by the composition

$$
\begin{aligned}
\left(\otimes^{l} s o(2 m, k)\right)_{S O(2 m, k)}^{-} \otimes & \left(\otimes^{r} s o(2 n, k)\right)_{S O(2 n, k)}^{-} \\
\rightarrow\left(\otimes^{l+r}\right. & s o(2 m, k) \times s o(2 n, k))_{S O(2 m, k) \times S O(2 n, k)}^{-} \\
& \rightarrow\left(\otimes^{l+r} s o(2(m+n), k)\right)_{S O(2(m+n), k)}^{-}
\end{aligned}
$$

Here the first map comes from the canonical inclusions $k^{2 m} \rightarrow k^{2 m} \times 0 \rightarrow k^{2(m+n)}$ and $k^{2 n} \rightarrow 0 \times k^{2 n} \rightarrow k^{2(m+n)}$, combined with the concatenation of tensors. The second map arises from the diagonal inclusion.

It is obvious that the direct sum

$$
\bigoplus_{m}\left(\otimes^{m} s o(2 m, k)\right)_{S O(2 m, k)}^{-}
$$

appears as a subalgebra of this algebra, which is isomorphic to the polynomial algebra $k[X]$.

4.4. Coming back to the group : $H_{3}(s o(4, A), k)$. It is implicit in [4] that the isomorphism

$$
H_{3}(s o(4, A), k)^{+} \stackrel{\cong}{\rightrightarrows} H D_{2}(A)
$$

is induced by the map

$$
\left(M_{1} \otimes M_{2} \otimes M_{3}\right) \otimes\left(a_{1} \otimes a_{2} \otimes a_{3}\right) \mapsto<\left[M_{1}, M_{2}\right], M_{3}>a_{1} \otimes a_{2} \otimes a_{3},
$$

where $\langle.,$.$\rangle is the Killing form.$

Similarly, one can show that the isomorphism

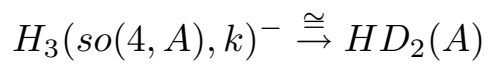


is induced by the map

$$
\left(M_{1} \otimes M_{2} \otimes M_{3}\right) \otimes\left(a_{1} \otimes a_{2} \otimes a_{3}\right) \mapsto \tilde{P f}\left(\left[M_{1}, M_{2}\right], M_{3}\right) a_{1} \otimes a_{2} \otimes a_{3} .
$$

\section{Acknowledgements}

I am grateful to J.-M. Lemaire for a stimulating discussion on the Pfaffian.

\section{References}

[1] A. Borel, Linear algebraic groups, second revised edition, Grad. Texts in Math. 126, Springer Verlag 1991.

[2] N. Bourbaki,Algèbre, chap. 9 : Formes sesquilinéaires et formes quadratiques, Hermann 1959.

[3] K.S. Brown, Cohomology of groups, Grad. Texts in Math. 87, Springer Verlag 1982.

[4] J.-L. Cathelineau, Homologie de degré trois d'algèbres de Lie simples déployées étendues à une algèbre commutative, L'Ens. Math. 33 (1987) 159-173.

[5] _ Projective configurations, Homology of Orthogonal groups and Milnor KTheory, Duke Math. J., 121 (2004) 343-387.

[6] _ Homology of Orthogonal groups : a quadratic algebra, K-Theory, 30 (2003) 13-35.

[7] Charney R.-M., A generalization of a theorem of Vogtmann, J. Pure Appl. Algebra 44 (1987) 107-125.

[8] C. Chevalley, S. Eilenberg, Cohomology theory of Lie groups and Lie algebras, Trans. Amer. Math. Soc., 63 (1948) 85-124.

[9] J.-C. Jantzen, Representations of algebraic groups, Pure and applied mathematics 131, Academic Press 1987.

[10] C. Kassel, Kähler differentials and coverings of complex simple Lie algebras extended over a commutative algebra, J. Pure Appl. Alg., 34 (1984) 265-275.

[11] J.-L. Koszul, Homologie et cohomologie des algèbres de Lie, Bull. Soc. Math. France, 78 (1950) 65-127.

[12] J.-L. Loday, Cyclic Homology, Grundl. Math. Wissen. 301, Springer Verlag Berlin, second edition 1998.

[13] _ Opérations sur l'homologie cyclique des algèbres commutatives, Inv. Math., 96 (1989) 205-230.

[14] - Procesi C., Homology of symplectic and orthogonal algebras, Adv. in Math., 69 (1988) 93-108.

[15] _ Quillen D., Cyclic homology and the Lie algebra homology of matrices, Comment. Math. Helv. 59 (1984) 565-591.

[16] J.-W. Milnor, Stasheff J.-D., Characteristic classes, Annals of Math. Studies 76, Princeton University Press 1974.

[17] B. Mirzaii, Van der Kallen W., Homology stability for unitary groups, Doc. Math. 7 (2002) 87-98.

[18] I. Panin, Homological stabilization for the orthogonal and symplectic groups, J. Soviet Math. 52 (1990) 3165-3170.

[19] C. Procesi, The invariant theory of $n \times n$-matrices, Adv. in Math., 19 (1976) 306-381.

[20] L.-N. Vaserstein, Stabilization of unitary and orthogonal groups over a ring with involution, Math USSR Sbornik, 10 (1970) 307-326.

[21] K. Vogtman, A Stiefel complex for the orthogonal group of a field, Comment. Math. Helvetici 57 (1982) 11-21.

Laboratoire Jean Dieudonné UMR CNRS 6621, Parc Valrose 06108 Nice Cedex 2

E-mail address: cathe@math.unice.fr 